\title{
Un gioco multimedia su argomenti di cultura e civiltà italiana
}

Adriana Pintori O livotto

\begin{abstract}
The lack of suitable didactic material for the study of the Italian Culture and Civilization, as well as the interest that this knowledge has for the complete acquisition of the I talian language, which does not only imply the knowledge of linguistic and grammar resources, as the traditional language teaching system would have thought, has made us decide to develop a multimedia game.

This computer game asks a series of questions which have different possible answers, and once the student answers, he gets visual and oral feeback on his answer, even if this answer is wrong.

The items presented are of general knowledge (history, geography, politics, well known institutions, literature, arts, science, show business and traditions). Therefore, the player acquires a knowledge similar to the one average I talians have.
\end{abstract}

O gni docente di lingue si rende conto fin dal primo momento del suo approccio con gli studenti del loro scarso conoscimento - salvo poche eccezioni della realtà cultural e straniera e della loro difficoltà per situarsi correttamente fra le due culture in gioco, quella propria e quella straniera. Se tralasciamo alcune nozioni artistiche (architettura, scultura, pittura), cinematografiche e folcloristiche, le conoscenze culturali dei nostri allievi sull'Italia sono realmente minime.

Siamo pienamente d'accordo con Vermeer (1991) quando afferma che la comunicazione è una cultura attualizzata, per cui è fondamentale l'acquisizione della civiltà e di tale cultura riferita al paese della lingua studiata: questa lingua, nuova per l'alunno, verrà considerata, quindi, come un insieme di processi pedagogici rivolti verso la padronanza dei componenti sociolinguistici e socioculturali di tale realtà culturale straniera attuale. Inoltre, nel caso di un futuro traduttore che dovrà fungere da mediatore e intermediario fra due culture diverse, dovremo approntare una strategia didattica specifica, tenendo conto del fenomeno culturale. Infatti, secondo N ida (1945):

Le parole non possono essere comprese correttamente se sono separate dai fenomeni culturali localizzati di cui esse sono il simbolo.

T ale strategia dovrà, quindi, necessariamente includere le strutture sociopolitiche, le ideologie preponderanti eil sistema etico e morale imperante. 
La conoscenza d'una cultura diversa permette allo studente di entrare in contatto con «mondi culturali estranei gli uni agli altri» (M ounin, 1963: 64). $\mathrm{E}$, attraverso il paragone fra le due culture e le domande che egli si pone, lo stimola perfino a conoscere e intendere in modo più completo quella propria: lo studio ragionato delle due culture gli offriranno la possibilità di comprendere e di maturare intellettualmente, grazie alla modifica, in determinati casi, delle proprie opinioni.

Come metodo didattico per risal tare le differenze socioculturali, possiamo ricorrere a esercizi comparativi di testi paralleli (il «turrón», come dolce imprescindibile in una festa natalizia spagnola, può essere presentato assieme al «panettone» italiano, ecc.) o a espressioni idiomatiche riferite alla cultura e che non abbiano equival ente nelle nostre due lingue.

In un primo momento lo studente assimila e «osserva» la cultura straniera attraverso il prisma deformato della propria; solo in un secondo tempo, e grazie al nostro intervento didattico oppure per conto proprio, sarà capace di rendersi conto dei meccanismi che determinano la somiglianza o la differenza tra le due culture; tali meccanismi sorgono come risultato dell'analisi comparativo-interculturale che il traduttore dovrà realizzare per trasmettere questo risultato al suo recettore meta.

Il valore dei componenti socioculturali dello studio della cultura di un determinato paese è lo stesso di quello dei fattori linguistici, logico-discorsivi e referenziali, motivo per il quale cercheremo di non ignorarli in un corso completo di lingua straniera.

Secondo Benucci-M icheli (1992: 202), per lo studente che:

$\mathrm{N}$ on si è formato sugli stessi condizionamenti extralinguistici, storici, sociali, che invece hanno segnato la personalità di parlanti nativi della corrispondente generazione, questi condizionamenti vanno creati «artificialmente» attraverso la riflessione e il confronto, in un'analisi contrastiva delle culture delle due lingue.

Consideriamo utile insistere su questo punto: per un adulto, studente d'una lingua straniera, si devono creare, in modo artificiale, questi condizionamenti, attraverso un'analisi contrastiva delle due culture, senza limitarsi unicamente agli aspetti fonologici e grammaticali tradizionali.

Superata la decade degli ottanta, si riconosce l'importanza di elementi della vita quotidiana, del modo di vivere, di vestirsi, d'alimentarsi e di pensare degli altri popoli, mentre fino a poco tempo prima erano considerati degni di essere insegnati solo gli aspetti «superiori» della civiltà, come possono esserlo la letteratura, l'arte, i sistemi filosofici; attualmente, quindi, come afferma D'Addio Colosimo (1985: 90-92), finalmente si è superata l'opinione che:

Insegnare una lingua straniera voglia dire insegnare la relativa grammatica insieme a un certo numero di parole. 
Limitarsi a questo studio della civiltà, implicita alla lingua, tornava a svantaggio di una civiltà esplicita; oggigiorno, tutti gli studiosi di questo campo sono pienamente coscienti dell'importanza fondamentale del fattore culturale nelle diverse fasi della traduzione, della natura bilingue e biculturale della loro attività professionale e del bisogno di formare esperti in comunicazione interculturale che rendano possibile la comprensione fra membri di diverse culture, in un concetto definito da T itone (1982: 189-192) di «identità plurilingue che elimini la barriera eretta fra le diverse culture».

G ià Beljaev (1968) teneva conto dell'importanza di tali elementi extralinguistici e affermava che:

II traduttore deve possedere una vasta preparazione politica e sociale, deve conoscere bene la storia, la geografia, I'economia, I'ordinamento politico, la cultura e la vita quotidiana del paese, e anche i diversi dialetti e la storia della lingua, deve avere familiarità con la letteratura.

Specialmente qualora si tratti di culture molto diverse, gli inevitabili riferimenti socioculturali generano parecchie difficoltà nel momento di trovarne le equivalenze in un'altra lingua, il che fa assumere una rilevanza particolarmente importante al rapporto fra lingua e società quando ci si riferisce alla traduzione d'un testo letterario.

M ounin (1963: 236-239) sosteneva che per ottenere una buona traduzione è indispensabile che sussistano due condizioni necessarie e non sufficienti indipendentemente, tali come lo studio della lingua straniera e lo studio sistematico dell'etnografia della comunità di cui tale lingua è espressione: senza questa doppia condizione, nessuna traduzione sarà soddisfacente e il traduttore in questione sarà sempre un traduttore incompleto.

$\mathrm{M}$ a che cosa si intende per civiltà, che cosa si deve insegnare, e in quale modo?

È evidente che alcuni elementi della realtà extralinguistica della cultura sono noti universalmente: quasi nessuno straniero ignora il significato della parola spagnola «corrida» 0 dell'italiana «pizza»; gli elementi sconosciuti devono essere presentati all'alunno, tenendo in conto che, con la migliore conoscenza mutua, alcuni diventeranno conosciuti; oggigiorno, tutti intendono il significato del vocabolo russo «perestroika».

La nostra affermazione, comunque non deve farci cadere nel pericolo dei «clichés» o delle immagini deformate da giudizi banali come i luoghi comuni, che vengono attribuiti agli abitanti di un paese nel loro aspetto più aneddotico.

U no dei primi inconvenienti che presenta lo studio della civiltà italiana è lo scarso materiale didattico offerto dalle case editrici al professore che non dispone di un testo unico che lo aiuti nella sua docenza; infatti, si pretende offrire una visione generale del mondo culturale straniero che comprenda tutti i campi, ma i testi che troviamo sono o troppo specializzati o troppo elementari (a livello di adolescenti enon servono per i nostri propositi). Inoltre, rappresentano un carico culturale troppo ampio per essere acquisiti in poco tempo. 
II grande aiuto offerto dalle nuove tecnologie, assieme all'infrastruttura della Facoltà di Traduttori e Interpreti dell'U niversitat Autònoma de BarceIona, ci ha stimolati, dinanzi a questa mancanza di materiale, ad elaborare un gioco multimedia intorno ad argomenti di cultura e civiltà italiana nel quale il computer propone una serie di domande con diverse possibilità di risposta. Lo studente riceve informazione in immagine e suono anche nelle risposte sbagliate.

Gli argomenti che riteniamo possano essere degni di studio saranno di storia e politica, geografia, istituzioni, arte e letteratura, scienza, folclore e spettacolo; consideriamo che il grado di conoscenze dello studente straniero debba essere simile a quello che possiedono i cittadini di cultura media di quel paese.

All'inizio di questo gioco di autoapprendimento, il giocatore può scegliere l'argomento che preferisce (storia e politica, sport e folclore, turismo e geografia, spettacolo, arte e letteratura, scienza e natura) e svolgerlo fino alla fine delle domande, o può passare a un capitolo in cui ci sono domande su ognuno degli argomenti. Se lo studente risponde correttamente subito, non può passare direttamente a un'altra domanda, ma deve leggere anche le indicazioni delle risposte sbagliate, perché anche da esse potrà ricevere informazioni utili, in modo da poter ricavare qualche nozione pure in questa circostanza.

Q ualche domanda presenta solo un testo scritto, altre possono essere completate da carte geografiche, (quando si riferiscono a argomenti di geografia o turismo) o corredate da fotografie, se si tratta di personaggi, ecc.

D omanda:

II nome del Premio N obel di medicina dell'anno 1988 è:

1. Tina Anselmi

2. Rita Levi M ontalcini

3. Franca Rame

Risposta:

1. N o, è stata la prima donna M inistro della Repubblica

2. Bene, ha studiato il fattore di crescita del sistema nervoso

3. $\mathrm{N} 0$, è un'attrice teatrale

(Q uesta domanda viene accompagnata dalla fotografia di R ita Levi M ontalcini)

$\mathrm{N}$ on abbiamo poi dimenticato argomenti d'arte (pittura, scultura, architettura) con le corrispondenti immagini.

Q uando ci si riferisce a poesie considerate note a ogni persona di cultura media nel paese d'origine, abbiamo creduto opportuno presentarne le frasi o i versi più famosi, scritti o recitati. M a abbiamo volutamente tralasciato argomenti di letteratura, perché li riteniamo degni di un'altra sede più seria ed approfondita; inoltre, la letteratura è una materia presente nei piani di studio della carriera scelta dai nostri studenti. 
U n'altra possibilità che offrono le nuove tecnologie è quella di includere brani di musica, colonne sonore o registrazioni di ogni genere alle domande 0 alle risposte, secondo quanto possa essere considerato più opportuno per una didattica, efficace per il professore e attraente per lo studente.

Una volta ultimato, questo gioco che è composto di circa seicento domande, registrato con il nome del suo autore, sarà considerato proprietà della U AB che potrà distribuirlo a chi ne faccia richiesta. D esidero specificare che questo tipo di materiale ha suscitato l'interesse di altri docenti di lingua della nostra Facoltà, per cui si sta già pensando di elaborarne due simili per i corsi di lingua araba e tedesca.

D opo aver superato gli ostacoli propri della scelta del sistema informatico più adatto al nostro scopo e dell'apprendimento di tale sistema per realizzare il nostro gioco, il rispetto dei diritti d'autore rappresenta uno dei punti più delicati per l'elaborazione di questo materiale didattico: l'impossibilità di poter utilizzare fotografie, registrazioni sonore o grafiche, video e così via, che non siano state effettuate dall'autore del gioco, senza dover ricorrere a materiale altrui che, evidentemente, dovrebbe essere rimunerato. Per un lavoro come questo che presentiamo, il bisogno di ricorrere a tali materiali è costante e la spesa che ciò rappresenterebbe sarebbe talmente elevata che I'U niversità non accetterebbe di farsene carico. Come esempio segnaliamo il fatto che una semplice cartolina illustrata rappresentativa di un'opera d'arte 0 anche turistica raggiunge una cifra di 15/20 mila pesetas, senza parlare del brano di una canzone e via dicendo.

Ad ogni modo, ci impegnamo a superare anche questo ostacolo al più presto, nella speranza che questo materiale didattico, che abbiamo già sperimentato con i nostri allievi durante le lezioni di Civiltà e Cultura italiana e che ha riscosso il loro entusiasmo, possa interessare anche altri docenti di italiano 0 altri centri in cui si impara tale lingua.

\section{Bibliografia}

BELJAEV, B.V. (1960). 0 cherki po psikologii obuchenia inostrannym yazykam, M oskvà: M ezhdunarodnaja Kniga. [T rad. italiana 1968. Saggi di psicologia dell'insegnamento delle lingue straniere. G enova: La N uova I talia.]

BENU CCI, A.; M ICHELI, P. (1992). «Progetto per l'organizzazione di seminari di traduzione integrativi dei corsi di lingua italiana per stranieri». Rassegna italiana di linguistica applicata, 1: 202.

D 'AdDio Colosim O, W . (1985). Lingua straniera e comunicazione: problemi di glottodidattica. Bologna: Zanichelli.

M OUN IN, G . (1963). Les problèmes théoriques de la traduction. Parigi: G allimard.

N IDA, E. (1945). «Linguistics and Ethnology in T raslation Problems». W ord, 1.

TITONE, R. (1982). «L'educazione plurilingue come superamento del concetto razzistico di identità culturale». Rivista italiana di linguistica applicata, XIV/2.

VermeER, H. (1991). Las teorías funcionales y sus implicaciones en la práctica y la didáctica. U niversidad de Las Palmas de G ran C anaria.

ZARATE, G. (1986). Enseigner une culture étrangère. Parigi: H achette. 